\title{
Smoke Detection Algorithm Based on Wavelet Transformation and Energy Analysis
}

\author{
Xu Yijun \\ School of Technology \\ Beijing Forestry University \\ Beijing, China, 15210507665 \\ xuyijunkg21@sina.com
}

\author{
HAN Ning \\ School of Technology \\ Beijing Forestry University \\ Beijing, China, 13501331829 \\ hn217@bjfu.com.cn
}

\begin{abstract}
A novel algorithm to detect the forest fire smoke based on the wavelet transformation and high-frequency energy analysis is proposed. The conventional color-based RGB algorithm has the difficulty to distinguish the smoke from other objects with the same color, such as clouds and fogs, so it leads to some false alarms. Facing the shortages of the RGB algorithm, we propose a wavelet-based algorithm to improve the detection method. After been transformed by the wavelet, the original picture will be divided into four sub-images. One of the subimages can reflect the low-frequency energy information of the original image and the other three can offer the high-frequency energy information of the original information in horizontal, vertical and diagonal directions correspondingly. Using the wavelet coefficients in the four sub-images, the energy value can be calculated. Through this way, the energy feature of the picture can be distracted and the energy parameters in frequency domain can be figured out. By comparing the ratios of high-frequency energy to the whole energy in the pictures of the smoke, cloud, and fog respectively, evident distinctions can be found. Thus, a special range of the smoke's high-frequency energy ratio can be set by large experimental data. By comparing the data obtained from the camera with the range for the smoke, we can make sure whether the object is smoke or something just with the similar color. The experimental results we obtained can accurately distinguish the smoke from the none-smoke images. Thus, this algorithm can improve the traditional color-based RGB algorithm and the false alarm rate can be reduced
\end{abstract}

Keywords-forest fire detection; RGB algorithm; Wavelet transformation; High-frequency energy ratio

\section{INTRODUCTION}

The conventional way to detect the forest fire is mainly based on the smoke detector sensors set in the outdoor field. They have some shortages to detect smoke in large outdoor areas. First, to detect the forest fire, they have to analyze some particles transported from the fire to the detector as smoke. Second, in order to cover all desired areas, several sensors needed to be applied and this is not cost-effective.

Therefore, the Video-based detection technology has been applied to the forest fire surveillance [2][8]. Its current performance is superior to those of traditional particle-sampling based detectors in terms of detection rate. But, when it comes to false alarm rate, it is still needs to be improved. The traditional image processing method to detect the forest fire is based on analyzing the color of the smoke in the picture through RGB algorithm[5], which can detect the changes of the pixels in the target areas[6][9]. Whereas if some substances with the same color as smokes, such as clouds and fog, appear in the scenes, the RGB algorithm cannot distinguish them from smokes and this has lead to relatively high false alarm rate.

Hence, we proposed a new algorithm to distinguish the smoke from the cloud and fog through the wavelet transformation and high-frequency energy ratio. The smoke, cloud, and fog display obvious distinctions in the highfrequency domain because of the differences in the airflow motion and diffusion caused by dissimilar chemical compositions and temperature in these substances. Besides, detecting the frequency of motion and diffusion in different materials can be accomplished by using the wavelet analysis [1]. With the wavelet coefficients distracted from the original pictures, the energy both in the high-frequency domain and the whole frequency domain can be calculated respectively [7][5]. By figuring out the ratio of high-frequency energy to the whole energy in the pictures, distinctive results within statistics law to differentiate these three substances can be discovered. Thus, the high-frequency energy ratio in each picture can make a distinction between smoke, cloud, and fog effectively. By this algorithm, the false alarm rate will be reduced dramatically.

\section{THEORY OF WAVELET TRANSFORMATION}

The wavelet model is first proposed by J.Morlet in 1974. After been improved by many scientists during the past years, the technology of wavelet analysis has been very mature. It provides a new powerful tool for the signal processing with the multi-resolution analysis and a good ability of timefrequency analysis and, thus, is known as mathematical microscope. In our world today, the wavelet has been applied into many fields of science like signal analysis, image processing, and quantum mechanics. Although the edge of the picture is generated by different reasons, when reflected in the basic components of the picture, it is the discontinuous point or the dramatically changing area at the gray level. This means the edge of the image is the high frequency part in the signal. Through the wavelet transformation, the high frequency information in the image can be extracted. Here is the principles and extraction process. 
For the two-dimensional discrete wavelet, there is one Two-dimensional scaling equation $\varphi(x, y)$ and three Twodimensional wavelet equations $\psi^{\mathrm{H}}(\mathrm{x}, \mathrm{y}), \psi^{\mathrm{V}}(\mathrm{x}, \mathrm{y})$, and $\Psi^{\mathrm{D}}(\mathrm{x}, \mathrm{y})$. Each of them is the product of the Onedimensional scaling equation $\varphi$ and the corresponding wavelet equation $\Psi$. Having excluded the results of onedimensional product, we get a separable scaling equation :

$$
\varphi(x, y)=\varphi(x) \varphi(y)
$$

and three separable wavelet equations of sensitive direction:

$$
\begin{aligned}
& \psi^{H}(x, y)=\psi(x, y) \varphi(x, y) \\
& \psi^{V}(x, y)=\psi(x, y) \varphi(x, y) \\
& \psi^{D}(x, y)=\psi(x, y) \varphi(x, y)
\end{aligned}
$$

These wavelet equations have changes in gray along the different directions: $\psi^{H}(x, y)$ changes along the column; $\psi^{V}(x, y)$ changes along the row; $\psi^{D}(x, y)$ changes along the diagonal direction.

The image can be viewed as a two-dimensional signal with different rows and columns. The color feature in the rows or columns can be regarded as a one-dimensional signal. Then, the two-dimensional wavelet transformation could be applied to the picture.

First, one-dimensional wavelet transformation was applied to the rows, then, the columns was down sampled with the rate of two. Second, the one-dimensional wavelet transformation was applied on columns the rows were down sampled with the rate of two. Finally, the original picture will be transformed into a one with four sub-images (Fig.1). Each one of the sub-images represents the information on the different directions of the picture in the frequency domain. As show in Fig.1, the wavelet sub-images LH, HL, HH possess the horizontal, vertical, and diagonal edges of the original picture.

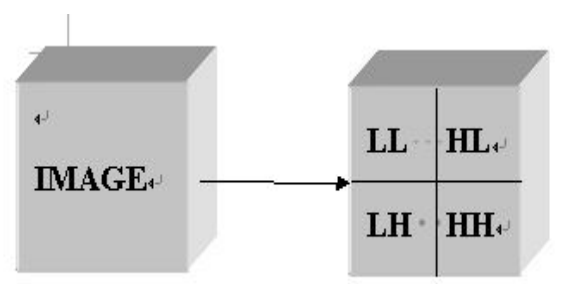

Figure1: Two-dimensional wavelet transformation of the image

\section{FOREST FIRE SMOKE DETECTION ALGORITHM}

The process of detecting the smoke is shown in the Fig.2. First, the RGB algorithm is used to analyze the image captured by the video camera set in the outside. Once the image is confirmed as the smoke, then further judge with the picture will be analyzed with the wavelet transformation algorithm.

Second, the target picture will be transformed by the wavelet algorithm. The original image would be transformed into one with four sub-images, which provide the information of the picture's edges in different directions.



Figure.2: The process of detecting the smoke

Second, the target picture will be transformed by the wavelet algorithm. The original image would be transformed into one with four sub-images, which provide the information of the picture's edges in different directions.

Third, with the information offered in the second step, we could figure out the high-frequency energy, total energy, and the high-frequency energy ratio in the images. Here are the equations:

$$
\begin{array}{r}
\text { High-frequency_energy }=\mathrm{V} * \mathrm{~V}+\mathrm{H} * \mathrm{H}+\mathrm{D} * \mathrm{D} \\
\text { Sum_energy }=\mathrm{C} * \mathrm{C}+\mathrm{V} * \mathrm{~V}+\mathrm{H} * \mathrm{H}+\mathrm{D} * \mathrm{D}
\end{array}
$$

High-frequency ratio

$$
\text { =High-frequency_energy/Sum_energy }
$$

Output the energy data High-frequency_energy and sum energy as "txt"file. We total the High-frequency energy and the sum energy of target picture. $\mathrm{V}, \mathrm{H}$, and $\mathrm{D}$ are all wavelet coefficient, the sum of there square reflects the energy value.

Fourth, we compare the high-frequency ratio value with the set value obtained from the experiments. If the highfrequency ratio value is in the smoke setting range, the picture is confirmed as the smoke image and the alarm is given. If the high-frequency ratio value is out of the range of the set value 
for the smoke. The possibility of the forest fire is excluded and the alarm will not be given.

\section{EXPERIMENTS}

\section{A. Experiments on the samples of the smoke, cloud and fog}

1) analysis of the smoke sample
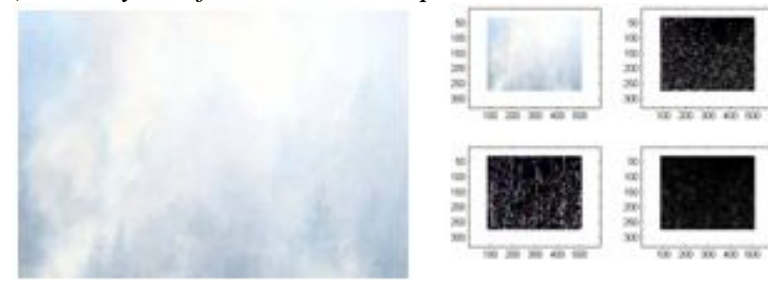

Figure3: The original picture and the transformed one

(5) (6) (7) can figure our the follow parameters: Highfrequency_energy, Sum_energy, and High-frequency energy ratio in the smoke sample.

TABLE1: SMOKE

\begin{tabular}{|c|c|c|c|}
\hline & $\begin{array}{c}\text { High- } \\
\text { frequency_energy }\end{array}$ & Sum_energy & $\begin{array}{c}\text { High-frequency } \\
\text { energy ratio }\end{array}$ \\
\hline Smoke & 112324 & $5.2370 \mathrm{e}+009$ & $2.1448 \mathrm{e}-005$ \\
\hline
\end{tabular}

\section{2) analysis of the cloud sample}
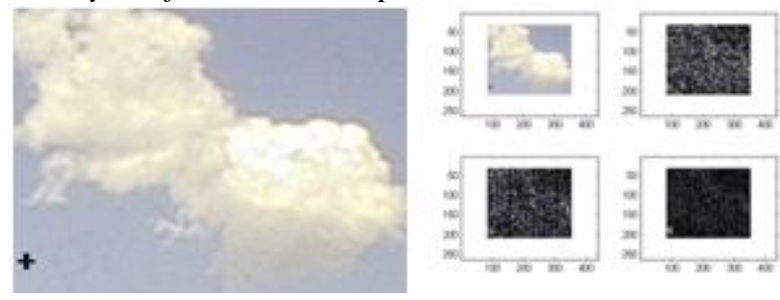

Figure4: The original picture and the transformed one

(5) (6) (7)can figure our the follow parameters: Highfrequency_energy, Sum_energy, High-frequency energy ratio in the cloud sample.

TABLE2: CLOUD

\begin{tabular}{|c|c|c|c|}
\hline & $\begin{array}{c}\text { High- } \\
\text { frequency_energy }\end{array}$ & Sum_energy & $\begin{array}{c}\text { High-frequency } \\
\text { energy ratio }\end{array}$ \\
\hline Cloud & 561810 & $6.8137 \mathrm{e}+009$ & $8.2453 \mathrm{e}-005$ \\
\hline
\end{tabular}

3) analysis of the fog sample

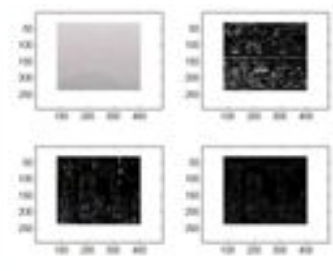

Figure5: The original picture and the transformed one

(5) (6) (7)can figure our the follow parameters: High- frequency_energy, Sum_energy, High-frequency energy ratio in the fog sample.

TABLE3: FOG

\begin{tabular}{|c|c|c|c|}
\hline & $\begin{array}{c}\text { High- } \\
\text { frequency_energy }\end{array}$ & Sum_energy & $\begin{array}{c}\text { High-frequency } \\
\text { energy ratio }\end{array}$ \\
\hline Fog & 45474 & $8.5281 \mathrm{e}+009$ & $5.3323 \mathrm{e}-006$ \\
\hline
\end{tabular}

From the above experiment, obvious distinctions appear in the high-frequency energy ratio in the samples of the smoke, cloud, and fog. Considering the fact that airflows, motions, diffusions, and chemical compositions in the three substances are different, we can make a prediction based on above experiment that the high-frequency energy ratio in these materials are dissimilar. Hence, based on further experiments, we can set a relatively certain range for every substance. The final range for the smoke can help us to make the alarm.

Then we repeated the experimental process described above on other samples of these three substances. The samples chose can reflect the diversity of these materials. Therefore, the experiment can be more rational.

\section{B. Results and Comparison}

TABLE4: EXPERIMENTAL RESULTS OF SMOKE, CLOUD, AND FOG

\begin{tabular}{|c|c|c|c|c|c|c|c|c|c|}
\hline $\begin{array}{l}\text { Sample } \\
\text { number }\end{array}$ & 1 & 2 & 3 & 4 & 5 & 6 & 7 & 8 & 9 \\
\hline \multirow[t]{2}{*}{ smoke } & $3.32 \mathrm{E}$ & $1.87 \mathrm{E}$ & $2.66 \mathrm{E}$ & $2.73 \mathrm{E}$ & $2.14 \mathrm{E}$ & $2.56 \mathrm{E}-$ & $3.01 \mathrm{E}-$ & $1.88 \mathrm{E}$ & $2.35 \mathrm{E}-$ \\
\hline & 05 & 05 & 05 & 05 & 05 & 05 & 05 & 05 & 05 \\
\hline \multirow[t]{2}{*}{ cloud } & $6.16 \mathrm{E}$ & $8.25 \mathrm{E}$ & $5.61 \mathrm{E}$ & $5.13 \mathrm{E}$ & $3.52 \mathrm{E}$ & $3.26 \mathrm{E}-$ & $4.43 \mathrm{E}-$ & $.73 \mathrm{E}$ & $4.33 \mathrm{E}-$ \\
\hline & 05 & 05 & 05 & 05 & 05 & 05 & 05 & 05 & 05 \\
\hline \multirow[t]{2}{*}{ fog } & $8.00 \mathrm{E}$ & $5.33 \mathrm{E}$ & $9.70 \mathrm{E}$ & $7.31 \mathrm{E}$ & $4.68 \mathrm{E}$ & $5.63 \mathrm{E}-$ & $1.01 \mathrm{E}-$ & $1.18 \mathrm{E}$ & $7.86 \mathrm{E}-$ \\
\hline & 06 & 06 & 06 & 06 & 06 & 06 & 05 & 05 & 06 \\
\hline
\end{tabular}

Collecting the data shown in the T4, the line graphs of these three substances can be drawn below in Fig6.

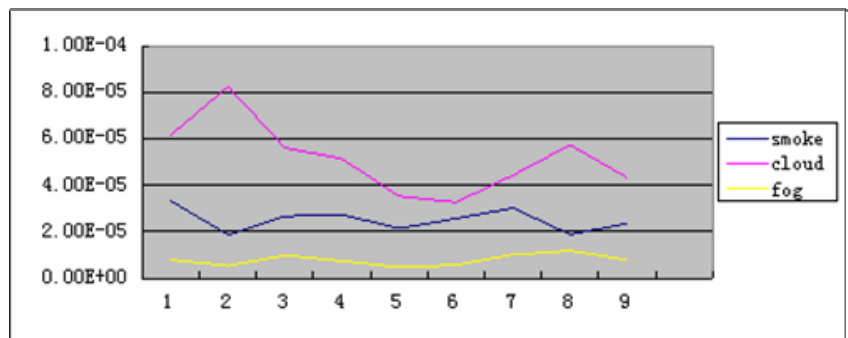

Figure6: The line graph of the ratios in smoke, cloud, and fog

From the data shown in the Fig6, we could find the highfrequency energy ratio in cloud is the larger than the other two. The range of the ratio in cloud is above $3.20 \mathrm{E}-05$. Besides, the high-frequency energy ratio in smoke is different from the other two and the range is between $1.80 \mathrm{E}-005$ and $3.5 \mathrm{E}-005$, with a very small overlap with the cloud range from the $3.20 \mathrm{E}-0.5$ to $3.50 \mathrm{E}-05$. What's more, the high-frequency energy ratio in fog is the lowest and the range is below $1.50 \mathrm{E}-$ 05 .

The comparison above indicts that the fog can be 
distinguished from the smoke perfectly. The cloud can be distinguished from the smoke in most cases. Although facing some special cloud, the method cannot totally distinguish it from the smoke, in most situations, the smoke can be detected effectively and the false alarm rate will be reduced dramatically. Thus, this wavelet-based algorithm to detect the smoke is a efficient one, which has improved the traditional RGB algorithm in terms of reducing the false alarm rate.

\section{CONCLUSION}

This paper proposes a wavelet-based algorithm of the forest smoke detection. The algorithm is proposed to solve the problem faced by the traditional color-based RGB algorithm. The RGB method fails to distinguish the smoke from the cloud and fog which share the same color with the smoke and, as a result, leads to a relatively high false alarm rate. By comparing the different high-frequency energy ratio in the smoke, cloud, and fog, we can effectively distinguish them and thus reduce the false alarm rate. Although this algorithm cannot differentiate the smoke with some special cloud, in most cases, it works well and the accuracy is high. In conclusion, the wavelet-based algorithm we proposed is a effective way to distinguish the smoke from the cloud and fog and to detect the forest fire.

\section{REFERENCE}

[1] Ali Rafiee,Reza Ta, vakoli Reza, Dianat Sara Abbaspour. Fire and Smoke Detection Using Wavelet. Analysis and Disorder Characteristics 978-1-61284-840-2/11 C2011 IEEE

[2] Wu Jiang, Huang Rule, Xu Ziyue, Han Ning.Forest Fire Smog Feature Extraction Based on Pulse-Coupled Neural Network. 978-1-4244-86250/11 @2011 IEEE

[3] Yuan Wei, Yu Chunyu, Zhang Yongming. Based on wavelet transformation fire smoke detection method 978-1-4244-3864-8/09 (C)2009 IEEE

[4] Yong-jun LIU, Cui-jian ZHAO, Su-jing SUN, Hong LI. Image Texture Recognition Method Research Based on Wavelet Technology 978-161284-449-7/11 C2011 IEEE

[5] Paolo Piccinini, Simone Calderara, Rita Cucchiara. Reliable Smoke Detection System In The Domains Of Image Energy And Color 978-14244-1764-3/08 (2008 IEEE 1376 ICIP

[6] Thou-Ho (Chao-Ho) Chen, Cheng-Liang Kao and Sju-Mo Chang.An Intelligent Real-Time Fire-Detection Method Based on Video Processing. 0-7803-7882-2/030002003 IEEE

[7] Anil Aksay, Alptekin Temizel CAMERA TAMPER DETECTION USING WAVELET ANALYSIS FOR VIDEO SURVEILLANCE 9781-4244-1696-7/07 (C2007 IEEE.

[8] Luo Qinjuan, Han Ning. Effective dynamic object detecting for videobased forest fire smog recognition, Proceedings of the 2009 2nd International congress on Image and Signal Processing, CISP 09,2009

[9] R. Gonzalez-Gonzalez, V. Alarcon-Aquino, R. Rosas-Romero, O. Starostenko, J. Rodriguez-Asomoza.Wavelet-Based Smoke Detection in Outdoor Video Sequences 978-1-4244-7773-9/10@2010 IEEE 